\title{
Fachsprachliche Vielfalt im Gegenwartsdeutschen
}

\author{
Diversity of Languages for Special Purposes \\ in Contemporary German
}

\begin{abstract}
ZUSAMMENFASSUNG
Die berufliche Kommunikation im deutschen Sprachraum ist in dreifacher Hinsicht durch Vielfalt geprägt: Zum einen besteht in diesem Bereich eine innere Mehrsprachigkeit hinsichtlich verschiedener Fachsprachen, die innerhalb einzelner Berufe verwendet werden. Zum anderen ist hier zunehmend eine äußere Mehrsprachigkeit zu beobachten, da viele Berufstätige das Deutsche nicht als erste, sondern als zweite bzw. fremde Sprache gebrauchen und darüber hinaus das Englische als internationale Lingua franca Verwendung findet. Und nicht zuletzt zeigt die berufliche Kommunikation in deutscher Sprache mit Differenzierung, Dezentralisierung und Dynamisierung drei wichtige Entwicklungstendenzen, die zu einem Ausbau der fachsprachlichen Vielfalt in vielen beruflichen Bereichen beitragen. Der vorliegende Beitrag greift innere und äußere Mehrsprachigkeit sowie das Triple-D fachsprachlicher Vielfalt im Gegenwartsdeutschen auf und weist auf sprachpolitische und -didaktische Konsequenzen hin, die sich hieraus ergeben. Schlüsselwörter: Fachsprachen, Sprachvielfalt, Mehrsprachigkeit
\end{abstract}

\section{ABSTRACT}

In the German speaking countries professional Communication shows diversity from three points of view: 1. internal multilingualism in relation to some languages for special purposes; 2 . external multilingualism in relation to German and/or English as a foreign language; 3. tendency of differentiation, decentralization, and dynamization. The article shows their connections as well as their political and didactic implications.

Keywords: languages for specific purposes, diversity of langauges, multilingualism

\section{Einleitende Bemerkungen}

Die berufliche Kommunikation im deutschen Sprachraum ist in dreifacher Hinsicht durch Vielfalt geprägt: Zum einen besteht in diesem Bereich eine innere Mehrsprachigkeit hinsichtlich verschiedener Fachsprachen, die innerhalb einzelner Berufe verwendet werden. Zum anderen ist hier zunehmend eine äußere Mehrsprachigkeit zu beobachten, da viele Berufstätige das Deutsche nicht als erste, sondern als zweite bzw. fremde Sprache gebrauchen und darüber hinaus das Englische als internationale Lingua franca Verwendung findet. Und nicht zuletzt

Thorsten Roelcke, Institut für Sprache und Kommunikation, Deutsch als Fremd- und Fachsprache, Technische Universität Berlin, Sekr.: HBS 2, Hardenbergstr. 16-18, 10623 Berlin, roelcke@tu-berlin.de, https://orcid.org/0000-0002-3894-6075 
zeigt die berufliche Kommunikation in deutscher Sprache mit Differenzierung, Dezentralisierung und Dynamisierung drei wichtige Entwicklungstendenzen, die zu einem Ausbau der fachsprachlichen Vielfalt in vielen beruflichen Bereichen beitragen. Der vorliegende Beitrag greift innere und äußere Mehrsprachigkeit sowie das Triple-D fachsprachlicher Vielfalt im Gegenwartsdeutschen auf und weist auf sprachpolitische und -didaktische Konsequenzen hin, die sich hieraus ergeben.

\section{Verschiedene Fächer}

Nach Henne (1986, S. 218) besteht „innere Mehrsprachigkeit“ im Sinne innerer Vielsprachigkeit einer ganzen Gesellschaft in einem verbreiteten Nebeneinander von verschiedenen sprachlichen Varietäten bzw. Registern des Deutschen. Dabei ist neben der Standardsprache, der Umgangssprache und den verschiedenen Mundarten insbesondere auch an wissenschaftliche, technische und institutionelle Fachsprachen zu denken. Die Frage, wie viele solcher Fachsprachen im deutschen Sprachraum bestehen und welche dies im Einzelnen sind, lässt sich kaum beantworten und hängt in starkem Maße davon ab, wie einzelne Fächer bestimmt und voneinander abgegrenzt werden. Als Richtschnur können hier institutionelle Fächereinteilungen dienen, deren Gliederungen nicht allein einzelne Fächer, sondern auch deren entsprechende Fachsprachen und kommunikative Bereiche definieren.

Zwei weit verbreitete fachliche bzw. berufliche Gliederungen aus dem deutschsprachigen Raum gehen aus dem „Verzeichnis der anerkannten Ausbildungsberufe" des Bundesinstituts für Berufsbildung (BIBB, 2000) und der „Systematik der Fächer und Fachkollegien“ der Deutschen Forschungsgemeinschaft (DFG) hervor: So beläuft sich die Gesamtzahl der Ausbildungsberufe im Jahr 2020 nach den Angaben des Bundesinstituts auf317 (zu deren Sprache und Kommunikation vgl. auch Efing \& Kiefer, 2018), die sich in 35 sog. Berufshauptgruppen untergliedern (vgl. Tab. 1). Die Zahl der wissenschaftlichen Fächer beträgt nach Angaben der Fachsystematik der DFG zusammen 213; diese werden in 49 „Fachkollegien“ zusammengefasst, die ihrerseits 14 größeren „Fachgebieten“ und vier „Wissenschaftsbereichen“ zugeordnet werden (vgl. Tab. 2). 
Tabelle 1. Ausbildungsberufe: Berufshauptgruppen (Angaben nach BIBB, 2020)

\begin{tabular}{|c|c|}
\hline Nr. & Berufshauptgruppe \\
\hline 11 & Land-, Tier- und Forstwirtschaftsberufe \\
\hline 12 & Gartenbauberufe und Floristik \\
\hline 21 & Rohstoffgewinnung und -aufbereitung, Glas- und Keramikherstellung und -verarbeitung \\
\hline 22 & Kunststoffherstellung und -verarbeitung, Holzbe- und -verarbeitung \\
\hline 23 & Papier- und Druckberufe, technische Mediengestaltung \\
\hline 24 & Metallerzeugung und -bearbeitung, Metallbauberufe \\
\hline 25 & Maschinen und Fahrzeugtechnikberufe \\
\hline 26 & Mechatronik-, Energie- und Elektroberufe \\
\hline 27 & $\begin{array}{l}\text { Technische Forschungs-, Entwicklungs-, Konstruktions- und } \\
\text { Produktionssteuerungsberufe }\end{array}$ \\
\hline 28 & Textil- und Lederberufe \\
\hline 29 & Lebensmittelherstellung und -verarbeitung \\
\hline 31 & Bauplanungs-, Architektur- und Vermessungsberufe \\
\hline 32 & Hoch- und Tiefbauberufe \\
\hline 33 & (Innen-)Ausbauberufe \\
\hline 34 & Gebäude- und versorgungstechnische Berufe \\
\hline 41 & Mathematik-, Biologie-, Chemie- und Physikberufe \\
\hline 42 & Geologie-, Geografie- und Umweltschutzberufe \\
\hline 43 & Informatik-, Informations- und Kommunikationstechnologieberufe \\
\hline 51 & Verkehrs- und Logistikberufe (außer Fahrzeugführung) \\
\hline 52 & Führer/innen von Fahrzeug- und Transportgeräten \\
\hline 53 & Schutz-, Sicherheits- und Überwachungsberufe \\
\hline 54 & Reinigungsberufe \\
\hline 61 & Einkaufs-, Vertriebs- und Handelsberufe \\
\hline 62 & Verkaufsberufe \\
\hline 63 & Tourismus-, Hotel- und Gaststättenberufe \\
\hline 71 & Berufe in Unternehmensführung und -organisation \\
\hline 72 & Berufe in Finanzdienstleistungen, Rechnungswesen und Steuerberatung \\
\hline 73 & Berufe in Recht und Verwaltung \\
\hline 81 & Medizinische Gesundheitsberufe \\
\hline 82 & Nichtmedizinische Gesundheits-, Körperpflege- und Wellnessberufe, Medizintechnik \\
\hline 83 & Erziehung, soziale und hauswirtschaftliche Berufe, Theologie \\
\hline 91 & Sprach-, literatur-, geistes-, gesellschafts- und wirtschaftswissenschaftliche Berufe \\
\hline 92 & Werbung, Marketing, kaufmännische und redaktionelle Medienberufe \\
\hline 93 & Produktdesign und kunsthandwerkliche Berufe, bildende Kunst, Musikinstrumentenbau \\
\hline 94 & Darstellende und unterhaltende Berufe \\
\hline
\end{tabular}


Tabelle 2. Wissenschaftliche Disziplinen: Fachkollegien (Angaben nach DFG, 2020)

\begin{tabular}{|c|c|}
\hline Nr. & Fachkollegium \\
\hline & Geistes- und Sozialwissenschaften \\
\hline 101 & Alte Kulturen \\
\hline 102 & Geschichtswissenschaften \\
\hline 103 & Kunst-, Musik-, Theater- und Medienwissenschaften \\
\hline 104 & Sprachwissenschaften \\
\hline 105 & Literaturwissenschaft \\
\hline 106 & $\begin{array}{l}\text { Sozial- und Kulturanthropologie, Außereuropäische Kulturen, Judaistik und } \\
\text { Religionswissenschaft }\end{array}$ \\
\hline 107 & Theologie \\
\hline 108 & Philosophie \\
\hline 109 & Erziehungswissenschaft und Bildungsforschung \\
\hline 110 & Psychologie \\
\hline 111 & Sozialwissenschaften \\
\hline 112 & Wirtschaftswissenschaften \\
\hline 113 & Rechtswissenschaften \\
\hline & Lebenswissenschaften \\
\hline 201 & Grundlagen der Biologie und Medizin \\
\hline 202 & Pflanzenwissenschaften \\
\hline 203 & Zoologie \\
\hline 204 & Mikrobiologie, Virologie und Immunologie \\
\hline 205 & Medizin \\
\hline 206 & Neurowissenschaften \\
\hline 207 & Agrar-, Forstwissenschaften und Tiermedizin \\
\hline & Naturwissenschaften \\
\hline 307 & Physik der kondensierten Materie \\
\hline 308 & Optik, Quantenoptik und Physik der Atome, Moleküle und Plasmen \\
\hline 309 & Teilchen, Kerne und Felder \\
\hline 310 & Statistische Physik, Weiche Materie, Biologische Physik, Nichtlineare Dynamik \\
\hline 311 & Astrophysik und Astronomie \\
\hline 312 & Mathematik \\
\hline 313 & Atmosphären-, Meeres- und Klimaforschung \\
\hline 314 & Geologie und Paläontologie \\
\hline 315 & Geophysik und Geodäsie \\
\hline 316 & Mineralogie, Petrologie und Geochemie \\
\hline 317 & Geographie \\
\hline 318 & Wasserforschung \\
\hline
\end{tabular}


Tabelle 2. Fortsetzung

\begin{tabular}{|c|l|}
\hline 321 & Molekülchemie \\
322 & Chemische Festkörper- und Oberflächenforschung \\
323 & Physikalische Chemie \\
324 & Analytische Chemie \\
325 & Biologische Chemie und Lebensmittelchemie \\
326 & Polymerforschung \\
327 & Theoretische Chemie \\
\hline & Ingenieurwissenschaften \\
401 & Produktionstechnik \\
402 & Mechanik und Konstruktiver Maschinenbau \\
403 & Verfahrenstechnik, Technische Chemie \\
404 & Strömungsmechanik, Technische Thermodynamik und Thermische Energietechnik \\
405 & Werkstofftechnik \\
406 & Materialwissenschaft \\
407 & Systemtechnik \\
408 & Elektrotechnik und Informationstechnik \\
409 & Informatik \\
410 & Bauwesen und Architektur \\
\hline
\end{tabular}

DieAnnahme, dass zujedemberuflichenBereichoderzujederwissenschaftlichen Disziplin eine eigene Fachsprache gehört, ist allerdings verkürzend: Zum einen können sich die fachsprachlichen Elemente einzelner Berufe und Disziplinen mehr oder weniger stark überschneiden (so etwa in den technischen Berufen oder in den Geisteswissenschaften), und zum anderen werden in einzelnen Berufen und Disziplinen in der Regel neben der deutschen Standardsprache mehrere verschiedene Fachsprachen und nicht nur eine Fachsprache verwendet (in der Praxis eines niedergelassenen Arztes oder seiner Kollegin neben derjenigen der betreffenden medizinischen Disziplin auch die des Rechts und der Verwaltung oder der Elektronischen Datenverarbeitung). Daher erlauben die Angaben nach BIBB und DFG lediglich eine grobe, aber immerhin doch institutionell verankerte Orientierung über die fachsprachliche Vielfalt des Deutschen.

Der Hinweis, dass berufliche (wie wissenschaftliche) Kommunikation meist nicht auf eine, sondern auf mehrere Fachsprachen zurückgreift, führt von der inneren Vielsprachigkeit fachlicher Kommunikation innerhalb des deutschen Sprachraums zur inneren Mehrsprachigkeit einzelner Personen, die in diesem Raum und dessen Gesellschaft beruflich (oder wissenschaftlich) handeln: Berufliche Kommunikation setzt nicht die Kenntnis einer einzelnen Fachsprache und im Allgemeinen die Fähigkeit, diese in Wort und Schrift angemessen 
$\mathrm{zu}$ verwenden, voraus. Vielmehr ist davon auszugehen, dass hier neben der Allgemein- oder Standardsprache und so etwas wie einer fächerübergreifenden Bildungssprache (in bestimmten Bereichen auch die ortsübliche Mundart) jeweils mehrere verschiedene Fachsprachen verwendet werden (vgl. Abb. 1, in der neben den sprachlichen Varietäten beruflicher Kommunikation auch die im betreffenden Beruf kommunizierenden Personen, deren Texte sowie sprachliche Ko- und nichtsprachliche Kontexte modelliert werden); folglich sollte auch von dem Terminus Berufssprache Abstand genommen und zwischen fachlicher Sprache bzw. Fachsprache und beruflicher Kommunikation bzw. Berufskommunikation unterschieden werden (vgl. Roelcke, 2020a).

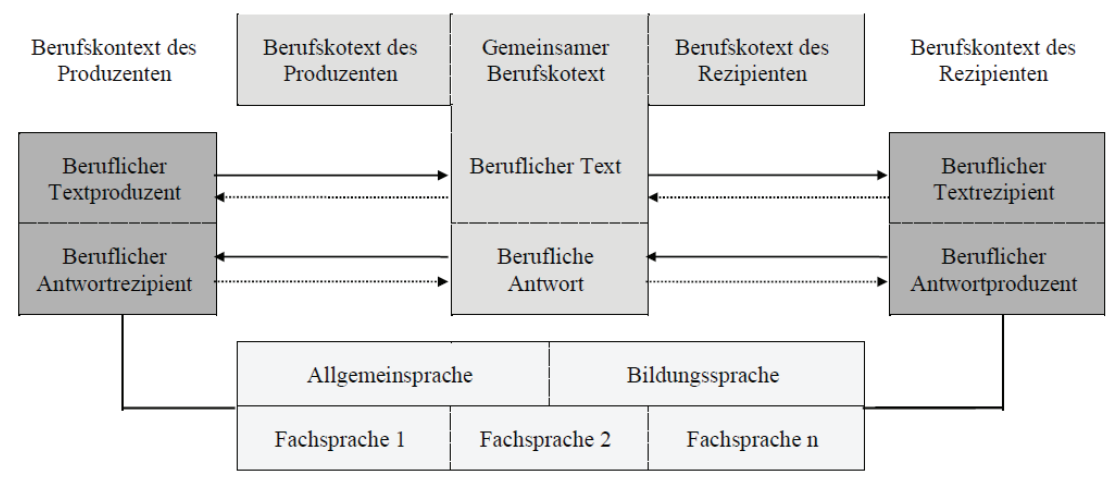

Abbildung 1: Modell beruflicher Kommunikation (Roelcke, 2020b, S. 21)

\section{Einzelne Fächer}

Neben der sprachlichen Vielfalt innerhalb der Kommunikation in verschiedenen Fächern (auf der sog. horizontalen Ebene) besteht im Gegenwartsdeutschen auch eine erhebliche Sprachvielfalt innerhalb einzelner Fächer selbst (auf der sog. vertikalen Ebene). Empirische Erhebungen oder institutionelle Einteilungen liegen hier nur in einem geringeren Umfang vor.

Die am besten ausgearbeitete Klassifikation stammt dabei von Lothar Hoffmann (1985, S. 64-70), der fünf Ebenen wissenschaftlich-technischer Fachsprachen unterscheidet und ihnen jeweils spezifische sprachlich-strukturelle sowie kommunikativ-pragmatische Merkmale zuweist (vgl. Tab. 3). Dabei erscheint der Ansatz der sprachlichen Merkmale aus systematischer Sicht stringent, da er in Stufen eine Abnahme von Elementen und Relationen natürlicher und eine Zunahme von solchen künstlicher Systeme vorsieht. Demgegenüber macht die Unterscheidung kommunikativer Merkmale einen weniger stringenten Eindruck, als hier auf den verschiedenen Ebenen jeweils mehrere unterschiedliche Szenarien genannt werden. Hierfür können mindestens zwei Gründe verantwortlich gemacht werden: Zum einen handelt es sich um eine echte Klassifikation, in der 
bestehende kommunikative Verhältnisse anhand von zwei singulären Kriterien eingeteilt werden; zum anderen ist die Kommunikation im wissenschaftlichtechnischen Bereich derart komplex, dass sie anhand dieser fünf Ebenen nicht hinreichend genau erfasst werden kann (hinsichtlich der Differenzierung vertikaler Gliederungsversuche vgl. auch die Kritik von Grucza, 2012, S. 125-131).

Tabelle 3. Ebenen fachlicher Kommunikation (nach Hoffmann, 1985, S. 64-70; vgl. hierzu auch Roelcke, 2020b, S. 50).

\begin{tabular}{|c|c|c|}
\hline & sprachliche Merkmale & kommunikative Merkmale \\
\hline $\begin{array}{l}\text { Sprache der theoretischen } \\
\text { Grundlagenwissenschaften } \\
\text { Sprache der experimentellen }\end{array}$ & $\begin{array}{l}\text { künstliche Symbole für } \\
\text { Elemente und Relationen }\end{array}$ & $\begin{array}{l}\text { Wissenschaftler } \\
\leftrightarrow \text { Wissenschaftler }\end{array}$ \\
\hline Wissenschaften & $\begin{array}{l}\text { künstliche Symbole für } \\
\text { Elemente; natürliche Sprache } \\
\text { für Relationen (Syntax) }\end{array}$ & $\begin{array}{l}\text { Wissenschaftler (Techniker) } \\
\leftrightarrow \text { Wissenschaftler } \\
\text { (Techniker) } \\
\leftrightarrow \text { wissenschaftlich- }\end{array}$ \\
\hline Sprache der angewandten & natürliche Sprache mit & technische Hilfskräfte \\
\hline Wissenschaften und der & einem sehr hohen Anteil an & Wissenschaftler (Techniker) \\
\hline Technik & $\begin{array}{l}\text { Fachterminologie und einer } \\
\text { streng determinierten Syntax }\end{array}$ & $\begin{array}{l}\leftrightarrow \text { wissenschaftliche und } \\
\text { technische Leiter der } \\
\text { materiellen Produktion }\end{array}$ \\
\hline Sprache der materiellen & natürliche Sprache mit & wissenschaftliche und \\
\hline Produktion & $\begin{array}{l}\text { einem hohen Anteil an } \\
\text { Fachterminologie und einer } \\
\text { relativ ungebundenen Syntax }\end{array}$ & $\begin{array}{l}\text { technische Leiter der } \\
\text { materiellen Produktion } \\
\leftrightarrow \text { Meister } \leftrightarrow \text { Facharbeiter } \\
\text { (Angestellte) }\end{array}$ \\
\hline Sprache der Konsumtion & $\begin{array}{l}\text { natürliche Sprache mit } \\
\text { einigen Fachtermini und } \\
\text { ungebundener Syntax }\end{array}$ & $\begin{array}{l}\text { Vertreter der materiellen } \\
\text { Produktion } \leftrightarrow \text { Vertreter des } \\
\text { Handels } \leftrightarrow \text { Konsumenten } \\
\leftrightarrow \text { Konsumenten }\end{array}$ \\
\hline
\end{tabular}

Angesichts der Komplexität von Kommunikation in Wissenschaft, Technik und Institutionen erscheint es letztlich mehr erfolgversprechend, statt einer Klassifikation eine Typologie anzusetzen, welche sich an der fachlichen Kompetenz bzw. Orientierung der Personen orientiert, die an der Kommunikation beteiligt sind, und dabei rekursiv angelegt ist, indem sie ein mehrfaches Erscheinen eines bestimmten Typs erlaubt. Eine solche Typologie unterscheidet etwa einen eher theoriebezogenen und einen eher anwendungsorientierten Bereich von fachlichen Experten sowie einen solchen von fachlichen Laien. Hiernach können dann fünf Typen der Kommunikation zwischen Experten und Laien unterschieden werden (Roelcke, 2014, S. 163-166):

- Typ 1 - Kommunikation unter Experten ein und desselben Faches: In diesem Fall findet die Kommunikation in einem einzelnen fachlichen Bereich statt, sie überschreitet also weder horizontale noch vertikale Grenzen. 
- Typ 2 - Kommunikation unter Laien in einem bestimmten Sachbereich: Die Kommunikation bezieht sich auf bestimmte Gegenstände und Sachverhalte und weist dabei keine fachliche Spezialisierung auf.

- Typ 3 - Kommunikation zwischen Experten verschiedener Ebenen bzw. Bereiche eines bestimmten Faches: Hier reicht die Kommunikation im Unterschied zu den ersten beiden Typen über die Grenzen zweier Ebenen oder Bereiche eines bestimmten Faches hinaus und wird von Experten geführt.

- Typ 4 - Kommunikation zwischen Experten eines bestimmten Faches und Laien im entsprechenden Sachbereich: Dieser Kommunikationstyp besteht ebenfalls nicht in einem Bereich alleine, sondern reicht von dem fachlichen Spezialbereich von Experten an den sachlichen Interessenbereichs von Laien heran.

- Typ 5 - Kommunikation zwischen Experten eines Faches und Experten eines anderen Faches: Dieser Typ besteht nicht über die Grenzen zweier Ebenen oder Bereiche ein und desselben Faches, sondern über die Grenzen zweier Fächer hinweg und ist somit mit Typ 4 vergleichbar, da hier Experten jeweils als Laien des anderen Faches erscheinen.

Diese Typologie erlaubt es nun, komplexe fachkommunikative Verhältnisse auf ganz verschiedenen vertikalen Ebenen zu erfassen und zu beschreiben. Dies ist bislang nur exemplarisch erfolgt (so zum Beispiel mit Blick auf die Sprache des Rechts; vgl. Roelcke 2017a), sodass umfassende Ergebnisse zur fachsprachlichen Vielfalt des Deutschen in einzelnen Fächern bzw. Berufen noch ausstehen. Hier besteht ein erhebliches fachsprachenlinguistisches Desiderat.

\section{Entwicklungstendenzen}

Die fachsprachliche Vielfalt im Deutschen zeigt sich nicht allein im Status quo innerer und äußerer Mehrsprachigkeit. Sie spiegelt sich darüber hinaus auch in drei Entwicklungstendenzen wider, welche den wachsenden sprachlichen Anforderungen und steigenden kommunikativen Herausforderungen entsprechen. Diese Tendenzen sind letztlich durch fachliche Entwicklungen selbst bedingt und können in Ausbildungs- wie in akademischen Berufen nachgewiesen werden (vgl. zum Folgenden Roelcke, 2017b) und lassen sich als Dynamisierung, Differenzierung und Dezentralisierung charakterisieren.

Die erste Entwicklungstendenz besteht in der Dynamisierung beruflicher Kommunikation und zeigt sich in der wachsenden Geschwindigkeit von deren Veränderungen. Letztlich geht diese Dynamisierung auf schnellere fachliche Entwicklungen selbst zurück und ist mit zunehmenden kommunikativen Herausforderungen verbunden. Die spiegelt sich zum Beispiel in den kurzen Intervallen wider, in denen seit dem Jahrhundertwechsel im Bundesgesetzblatt aktualisierte Ausbildungsverordnungen zum Kraftfahrzeugmechatroniker bzw. zur Kraftfahrzeugmechatronikerin erscheine: (vgl. die Ausgabejahre 1989, 2003, 2007, 2013 mit einem ersten Intervall von 14 und mehreren anschließenden Intervallen von jeweils wenigen Jahren). Analoge Entwicklungen sind auch im akademischen Bereich auszumachen, in dem einige Disziplinen wie etwa 
die Medizintechnik rasante Fortschritte erzielen. - Die Feststellung einer Dynamisierung fachlicher bzw. beruflicher Kommunikation darf indessen nicht mit einem naiven fortschrittsgläubigen Gesellschafts- oder Wissenschaftsbild verwechselt werden: Es bestehen neben dynamischen Bereichen auch solche, die durch eine Retardierung ihrer fachlichen (wie sprachlichen) Entwicklung geprägt sind; zu denken ist hier etwa an alte Handwerksberufe wie Gerber/Gerberin, Weber/Weberin oder Drahtzieher/Drahtzieherin, sofern diese nicht in neue Berufsbilder eingehen.

Differenzierung stellt die zweite Entwicklungstendenz der beruflichen Kommunikation dar. Sie zeigt sich in einer Bildung von ausspezialisierten fachsprachlichen Varianten, die der Entstehung eigener Teildisziplinen oder selbständiger Disziplinen folgt und jeweils spezifische sprachliche Anforderungen bzw. kommunikative Herausforderungen mit sich bringt. Auch diese Tendenz lässt sich anhand der Ausbildungsverordnungen zum Kraftfahrzeugmechatroniker bzw. zur Kraftfahrzeugmechatronikerin nachweisen: So ersetzt zwar die Verordnung aus dem Jahr2003 zwei ältere Verordnungen aus dem Jahr 1989(Kraftfahrzeugmechanik und Kraftfahrzeugelektrik), doch werden hier anstelle dieser beiden letztlich vier Schwerpunkte gesetzt, die im Jahr 2013 sogar um einen weiteren erweitert werden (Personenwagentechnik, Nutzfahrzeugtechnik, Motorradtechnik, System- und Hochvolttechnik sowie Karosserietechnik). Mit dieser fachlichen Differenzierung ist nun auch eine sprachliche Differenzierung mit verschiedenen kommunikativen Schwerpunkten verbunden, die neuen kommunikativen Anforderungen Rechnung trägt. Auch im akademischen Bereich sind zahlreiche fachliche Spezialisierungen im Sinne einer solchen Ausdifferenzierung festzustellen (zu denken ist hier etwa an zunehmende Spezialisierungen von Ärztinnen und Ärzten an Krankenhäusern) und gehen mit entsprechenden sprachlichen Veränderungen und kommunikativen Bedingungen bzw. Herausforderungen einher.

Unter der dritten Entwicklungstendenz, der Dezentralisierung von berufssprachlicher Kommunikation, ist eine Zunahme des Einflusses bzw. der Bedeutung von fachfremden Bereichen zu verstehen. Sie zeigt sich in einer vermehrten Wechselwirkung zwischen einzelnen fachlichen Bereichen, die ihrerseits weitere sprachliche Anforderungen bzw. kommunikative Herausforderungen mit sich bringt. Auch diese Dezentralisierung schlägt sich in den Ausbildungsverordnungen zum KraftfahrzeugmechatronikHandwerk nieder, indem hier neben der Vermittlung und dem Erwerb von fachlichen und sprachlichen Kenntnissen und Kompetenzen in den Bereichen Mechanik und Elektronik auch solche in Recht und Verwaltung, Elektronischer Datenverarbeitung und Kundenbetreuung vorgesehen sind. Im Bereich der Medizin ist eine entsprechende Entwicklung zu beobachten, indem etwa niedergelassene Ärztinnen und Ärzte der Allgemeinmedizin nicht allein zunehmend fachärztliche Kenntnisse und Kompetenzen nachweisen müssen, sondern darüber hinaus auch 
über weitreichende fachliche und kommunikative Fähigkeiten mit Blick auf Elektronische Datenverarbeitung, Personalmanagement, Rahmenvorgaben aus Recht und Verwaltung sowie Abrechnungswesen zu verfügen haben.

\section{Andere Sprachen}

Die fachsprachliche Vielfalt des Deutschen besteht also horizontal in einer Vielzahl spezifischer Fachsprachen diverser Fächer und vertikal in einer jeweils eigenen Zahl an kommunikativen Ebenen, auf denen hier kommuniziert werden kann; diese komplexe Situation ist darüber hinaus von einer Dynamisierung, Differenzierung und Dezentralisierung beruflicher Kommunikation geprägt. Personen, die am Berufsleben teilhaben, greifen in der Regel auf mehrere verschiedene Fachsprachen auf jeweils unterschiedlichen Ebenen zurück, sodass nicht der deutsche Sprachraum oder die deutschsprachige Gesellschaft allein durch fachsprachliche Vielfalt geprägt ist, sondern auch die fachliche bzw. sachliche Kommunikation einzelner Personen. Angesichts der sprachlichen Herkunft einzelner Personen greift dieser Befund jedoch zu kurz: Da Deutschland, Österreich und die Schweiz seit der Mitte des letzten Jahrhunderts jeweils mehr oder weniger als sog. Einwanderungsländer zu gelten haben, in denen Menschen leben, deren zuerst erworbene Sprache nicht das Deutsche, sondern eine andere Sprache wie Italienisch, Türkisch oder Arabisch ist, gilt es, hier zu differenzieren; hinzu kommt, dass nicht alleine international, sondern auch im deutschsprachigen Raum das Englische als Lingua franca Verwendung findet.

Vor diesem Hintergrund hat ein Aufriss der fachsprachlichen Vielfalt im Gegenwartsdeutschen auch dessen Status als erster oder fremder Sprache (auf die Unterscheidung zwischen Deutsch als Fremd- und Deutsch als Zweitsprache wird hier angesichts heterogener Spracherwerbsbiographien verzichtet) sowie die Konkurrenz oder Alternative durch den Gebrauch des Englischen zu berücksichtigen (zur fachlichen Mehrsprachigkeit vgl. auch die einschlägigen Abschnitte in Auer \& Wei, 2009 sowie Földes \& Roelcke, im Ersch.). Eine solche Verbindung der Konzepte innerer und äußerer Mehrsprachigkeit im Bereich fachlicher und beruflicher Kommunikation kann in einer Matrix erfolgen, in

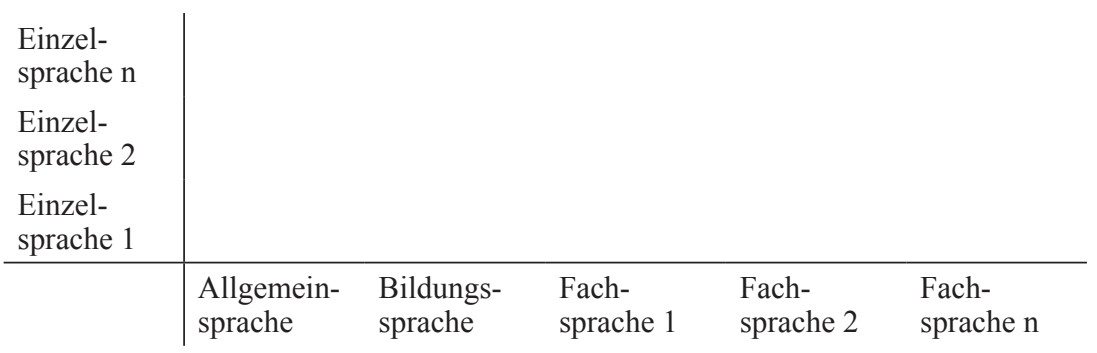

Abbildung 2: Äußere und innere Mehrsprachigkeit beruflicher Kommunikation (vgl. Roelcke, im Ersch.) 
der für jede einzelne Sprache eine Allgemein- und eine Bildungssprache sowie verschiedene Fachsprachen angesetzt werden (vgl. Abb. 2, in die noch keine konkreten Daten eingetragen sind): Dabei wird im Falle der fachsprachlichen Vielfalt des Deutschen davon ausgegangen, dass zum einen das Deutsche als eine der Einzelsprachen als erste oder als fremde Sprache erworben wurde und zum anderen die Kompetenzen in der Allgemein- und in der Bildungssprache sowie in verschiedenen Fachsprachen unterschiedlich stark ausgeprägt sind.

Auch diese Kombination äußerer und innerer Mehrsprachigkeit beruflicher Kommunikation lässt sich bislang nur exemplifizieren und nicht exhaustiv für einen bestimmten fachlichen oder beruflichen Bereich oder gar für den deutschen Sprachraum als Ganzes darstellen. Ein - geradezu stereotypes - Beispiel mag in diesem Zusammenhang eine Kraftfahrzeugwerkstatt in Berlin-Kreuzberg darstellen, in der neben Deutsch auch die Sprachen Türkisch und Englisch verwendet werden (vgl. Abb. 3). Dieses Szenario fachlicher Mehrsprachigkeit kann nun durch verschiedene Personen unterschiedlich abgedeckt werden: So ist zum einen ein Facharbeiter denkbar, der Deutsch als Erstsprache erworben und in dieser Sprache auch seine Ausbildung abgeschlossen hat; des Türkischen ist er weder allgemein- und bildungs- noch fachsprachlich mächtig, während er über

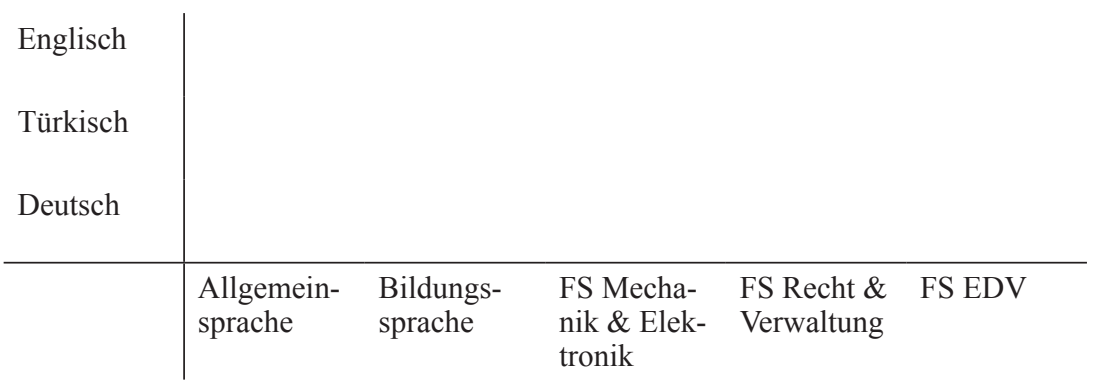

Abbildung 3: Äußere und innere Mehrsprachigkeit in einer Kfz-Werkstatt; FS = Fachsprache

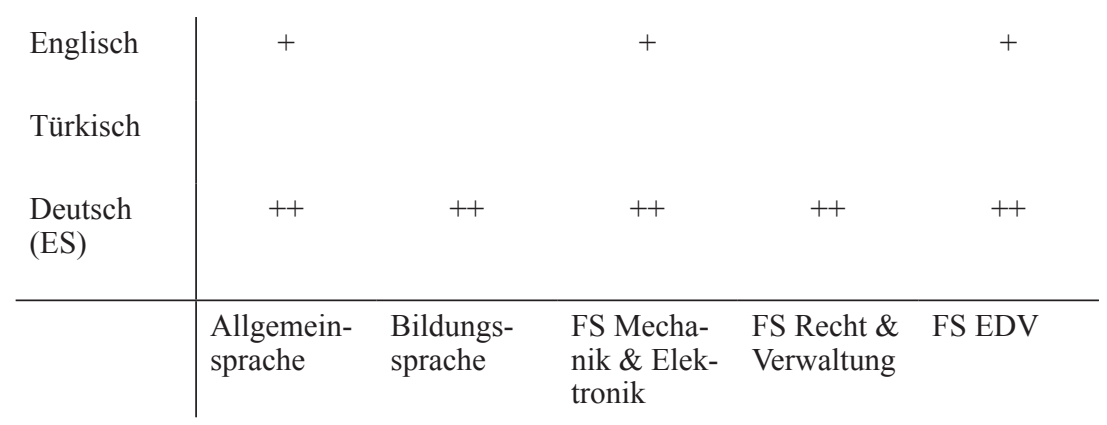

Abbildung 4: Mehrsprachigkeit einer Kfz-Werkstatt: Person 1; ES = Erstsprache, FS = Fachsprache 
Grundkenntnisse in der englischen Allgemeinsprache sowie in deren Fachsprachen der Mechanik und Elektronik und der Elektronischen Datenverarbeitung verfügt (vgl. Abb. 4). Zum anderen ist hier an eine Auszubildende zu denken, die Türkisch als erste und Deutsch als zweite Sprache erworben hat und dabei aufgrund ihrer Beschulung in Deutsch nur über eingeschränkte Kompetenzen in der türkischen Bildungssprache verfügt; ihre fachsprachlichen Kompetenzen sind derzeit noch im Aufbau begriffen - im Falle des Deutschen und des Englischen im Rahmen ihrer Ausbildung, im Falle des Türkischen durch den fachlichen Austausch mit Kolleginnen und Kollegen sowie Kundschaft der Werkstatt (vgl. Abb. 5).

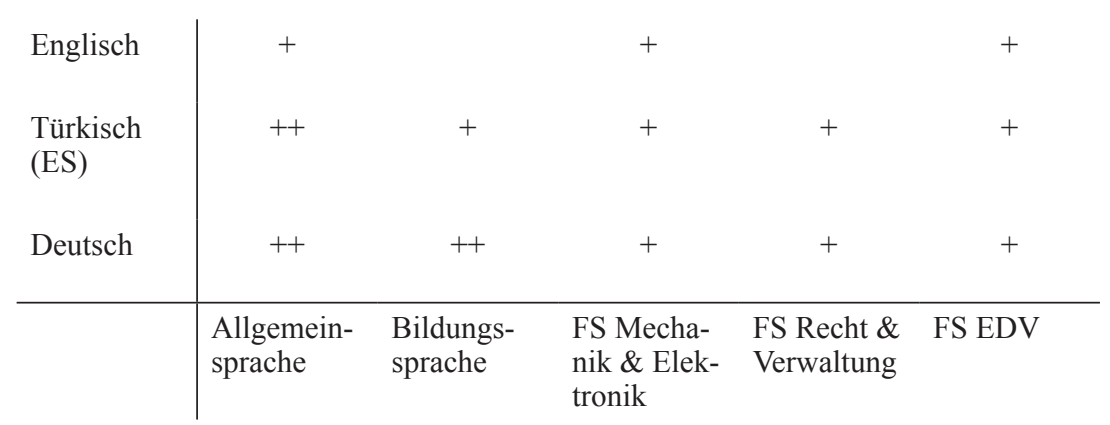

Abbildung 5: Mehrsprachigkeit einer Kfz-Werkstatt: Person 2; ES = Erstsprache, FS = Fachsprache

\section{Abschließende Bemerkungen}

Der vorliegende Abriss fachsprachlicher Vielfalt im Gegenwartsdeutschen lässt sich wie folgt zusammenfassen:

1) Der deutsche Sprachraum umfasst eine Vielzahl an verschiedenen Fachsprachen, die sich anhand von Fächergliederungen umreißen lässt; berufliche Kommunikation greift dabei in der Regel auf mehrere Fachsprachen zurück.

2) Innerhalb einzelner Fächer ist fachsprachliche Vielfalt durch verschiedene kommunikative Ebenen und Bereiche bedingt, die sich an unterschiedlicher fachlicher Expertise bis hin zum Laientum festmachen lassen.

3) Auch wenn einzelne Fächer und deren Fachsprachen im Verschwinden begriffen sind, lassen sich mit Dynamisierung, Differenzierung und Dezentralisierung drei Entwicklungstendenzen innerhalb der fachsprachlichen Vielfalt ausmachen.

4) Angesichts der Vielsprachigkeit im deutschen Sprachraum sind mehrsprachige Szenarios zu denken, die von verschiedenen Vertreterinnen und Vertretern der entsprechenden Fächer bzw. Berufe unterschiedlich abgedeckt werden.

Im Unterschied zum horizontalen Spektrum an Fachsprachen, das sich anhand von Fächer- bzw. Berufskanons zumindest indirekt erschließen lässt, liegen über 
deren vertikale Gliederungen und mehrsprachige Szenarios bisher kaum mehr als exemplarische Erkenntnisse vor - hier besteht ein erhebliches Desiderat für die Fachsprachenlinguistik bzw. Fachkommunikationsforschung. Dies gilt umso mehr, als weitere Erkenntnisse zur fachsprachlichen Vertikalität und Mehrsprachigkeit in der beruflichen Kommunikation eine wichtige Grundlage für künftige fachsprachendidaktische Vorhaben sowie für zielgerechte bildungs- und sprachpolitische Entscheidungen darstellen dürften.

\section{References}

Auer, P., \& Wei, L. (Eds.) (2009). Handbook of Multilingualism and Multicultural Communication. Berlin, New York: De Gruyter.

Bundesinstitut für Berufsbildung (2020). Verzeichnis der anerkannten Ausbildungsberufe 2020. Bonn. Retrieved February 19, 2021, from https://www.bibb.de/veroeffentlichungen/de/ publication/show/16754.

Deutsche Forschungsgemeinschaft (2020). Fachsystematik (Stand: 20. Oktober 2020). Bonn. Retrieved February 19, 2021, from https://www.dfg.de/dfg_profil/gremien/fachkollegien/ faecher/index.jsp.

Efing, Ch, \& Kiefer, K.-H. (Eds.) (2018). Sprache und Kommunikation in der beruflichen Aus- und Weiterbildung. Ein interdisziplinäres Handbuch. Tübingen: Narr.

Földes, C., \& Roelcke, Th. (Eds.) (in press). Handbuch Mehrsprachigkeit. Berlin, Boston: De Gruyter.

Grucza, S. (2012). Fachsprachenlinguistik. Frankfurt am Main: Peter Lang Verlag.

Henne, H. (1986). Jugend und ihre Sprache. Darstellung, Materialien, Kritik. Berlin, New York: De Gruyter.

Hoffmann, L. (1985). Kommunikationsmittel Fachsprache. Eine Einführung (2nd ed.). Tübingen: Narr.

Roelcke, Th. (2014). Zur Gliederung von Fachsprache und Fachkommunikation. Fachsprache. International Journal of Specialized Communication, 36(3-4), 154-178.

Roelcke, Th. (2017a). Rechtssprachliche Kommunikation. Eine Typologie. In J. Engberg, K. Luttermann, S. Cacchiani, \& C. Preite (Eds.), Popularization and Knowledge Mediation in the Law / Popularisierung und Wissensvermittlung im Recht (S. 3-27) Münster: Lit.

Roelcke, Th. (2017b). Dynamisierung - Differenzierung - Dezentralisierung. Tendenzen beruflicher Kommunikation im Deutschen am Beispiel der Verordnung über die Berufsausbildung zum Kraftfahrzeugmechatroniker und zur Kraftfahrzeugmechatronikerin. Glottotheory, 8(2), $155-170$.

Roelcke, Th. (2020a). Berufssprache und Berufliche Kommunikation - eine konzeptionelle Klärung. Sprache im Beruf, 3(1), 3-17.

Roelcke, Th. (2020b). Fachsprachen (4th. ed.). Berlin: Erich Schmidt Verlag

Roelcke, Th. (in press). Mehrsprachigkeit in der Fachkommunikation. In İ. Dirim, \& J. Meier (Eds.), Handbuch Mehrsprachigkeit. Interdisziplinäre Zugänge zu Mehrsprachigkeit und sozialer Teilhabe. Bad Heilbrunn: Klinkhardt. 